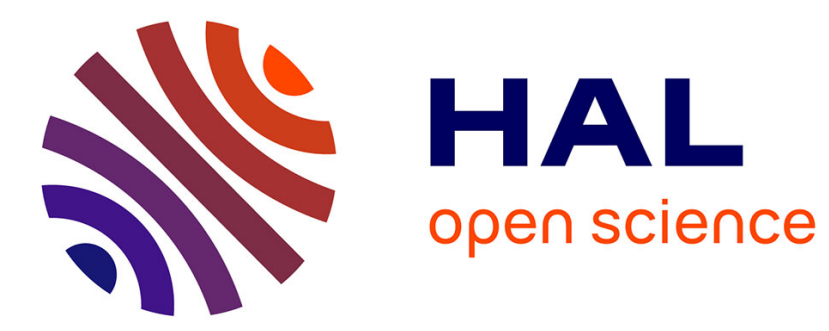

\title{
Problèmes théoriques (et pratiques) posés par la reconstruction du genre neutre en protoroman Eva Buchi, Yan Greub
}

\section{To cite this version:}

Eva Buchi, Yan Greub. Problèmes théoriques (et pratiques) posés par la reconstruction du genre neutre en protoroman. Éva Buchi; Wolfgang Schweickard. Dictionnaire Étymologique Roman (DÉRom) 2. Pratique lexicographique et réflexions théoriques, 402, De Gruyter, pp.67-78, 2016, Beihefte zur Zeitschrift für romanische Philologie, 978-3-11-045026-2. halshs-01421348

\section{HAL Id: halshs-01421348 https://shs.hal.science/halshs-01421348}

Submitted on 22 Dec 2016

HAL is a multi-disciplinary open access archive for the deposit and dissemination of scientific research documents, whether they are published or not. The documents may come from teaching and research institutions in France or abroad, or from public or private research centers.
L'archive ouverte pluridisciplinaire HAL, est destinée au dépôt et à la diffusion de documents scientifiques de niveau recherche, publiés ou non, émanant des établissements d'enseignement et de recherche français ou étrangers, des laboratoires publics ou privés. 

en protoroman

\section{Introduction}

Ce chapitre présente, dans une optique méta-lexicographique, mais pas seulement, le cas d'un problème théorique auquel l'équipe du Dictionnaire Étymologique Roman (DÉRom) a été et continue à être confrontée, et qui constitue, en réalité, une question classique de la linguistique romane (cf. Meyer-Lübke 1883). ${ }^{1}$

Le DÉRom a pour objectif de rebâtir l'étymologie du lexique héréditaire roman, et dans une première phase, celle du noyau commun de cet ensemble : les unités lexicales qui sont (apparemment) présentes dans tous les domaines romans. Nous avons jugé nécessaire (et c'est la raison d'être du projet) de le faire selon une méthode essentiellement différente de celle qu'avait utilisée MeyerLübke pour son Romanisches Etymologisches Wörterbuch $\left(\mathrm{REW}_{3}\right)$ : la reconstruction comparative (cf. par exemple Campbell 2013, 107-158 [" The Comparative Method and Linguistic Reconstruction »]). Elle n'est pas seulement différente de celle de ce dictionnaire, dont la dernière édition remonte à 1935, mais différente de la plus grande part des travaux qui se réalisent dans l'ensemble de ce qu'on appelle en allemand la Romanistik. En effet, la méthode de la reconstruction

1 Une première version de ce chapitre a été présentée dans la section «Linguistique latine/linguistique romane » du XXVII ${ }^{e}$ Congrès international de linguistique et de philologie romanes, qui s'est tenu du 15 au 20 juillet 2013 à Nancy. Nos remerciements les plus chaleureux s'adressent à tous les déromiens, en particulier à Victor Celac et Mihaela-Mariana Morcov (tous les deux Académie roumaine, Institut de linguistique «Iorgu Iordan - Al. Rosetti », Bucarest), à Wolfgang Schweickard (Université de la Sarre, Sarrebruck) et à Pierre Swiggers (FWO/Université de Leuven), qui ont bien voulu nous communiquer leurs réactions à notre texte, de même qu'aux congressistes, notamment à Marcello Barbato (Université de Naples - L'Orientale) et à Romain Garnier (Université de Limoges/IUF), qui ont pris part à la discussion suite à notre présentation orale.

Adresse de correspondance : Éva Buchi et Yan Greub, ATILF (CNRS/Université de Lorraine), B.P. 30687, F-54063 Nancy Cedex, eva.buchi@atilf.fr et yan.greub@atilf.fr. 
comparative, " ascendante », a toujours été jugée peu rentable dans le domaine des langues romanes, dont l'ancêtre commun - ou plus précisément un système linguistique proche de leur ancêtre commun - était très bien attesté, ce qui a semblé recommander la méthode « descendante».

Il y a quelque chose d'étonnant, si l'on veut y penser, à ce qu'une méthode si répandue, si classique, si bien installée, puisse donner lieu, par sa simple application à un niveau basique, à de sérieux problèmes théoriques. C'est pourtant l'expérience de la rédaction du DÉRom, et on peut a posteriori comprendre les causes de ces problèmes : c'est qu'en effet, la reconstruction linguistique est une idée neuve en romanistique. Et par conséquent, chaque occasion de son application mène le rédacteur dans un vide conceptuel, une carence bibliographique (la bibliographie est toujours très étendue, bien sûr, mais une part immense de ce qui est écrit ne peut servir, sinon avec de grands efforts d'adaptation), et situe ce rédacteur face à un grand trouble : celui de devoir sortir de ses schèmes de pensée les mieux établis. On comprend que la méthode entraîne le doute. Dans ce qui suit, nous voudrions présenter une de ces occasions d'hésitation, qui porte sur la reconstruction du genre neutre en protoroman.

\section{Historique de la réflexion au sein du DÉRom}

\subsection{Le degré zéro de la conceptualisation}

Dans un premier temps, l'équipe du DÉRom ne s'est guère posé de questions : partie de la romanistique, dépendante d'elle, elle adoptait ses conclusions sans bien réfléchir aux moyens qu'elle utilisait pour les atteindre. Nous prévoyions donc d'assigner la catégorie grammaticale neutre à des substatifs reconnus comme neutres par la tradition, celle de la romanistique comme celle des études latines. Les auteurs des articles du DÉRom, comme tout un chacun, allaient chercher la catégorie grammaticale de */'ali-u/, */'lakt-e/ ou */'mustu/ dans le TLL, heureusement confirmé par le $\mathrm{REW}_{3}$. Ils y trouvaient sinon la catégorie grammaticale elle-même (en principe, elle n'a jamais été attribuée, dans les articles du DÉRom, sans analyse critique), au moins son nom.

Lors du lancement du projet en janvier 2008, la nomenclature du DÉRom prévoyait, à côté de substantifs masculins et féminins, des substantifs étiquetés neutres comme */'ali-u/ s.n. (TLL 1, 1619: " ālium, vulgariter allium vel aleum, -īn. »; $\mathrm{REW}_{3}$ : « allium »), */'lakt-e/ s.n. (TLL 7/2, 814 : «lac (lāc ?), lactis n. »; $\mathrm{REW}_{3}$ : «lac [...], 2. *lacte ») ou */'must-u/ s.n. (TLL 8, 1712, s.v. mustus, -a, -um : 
« subst. mustum, -ī n. »; $\mathrm{REW}_{3}$ : «mŭstum »). Cette caractérisation en tant que neutres ne reposait sur aucune analyse menée au sein du projet, mais constituait au contraire un héritage de la tradition romanistique, qui reconnaît à l'ancêtre commun des langues romanes, appelé en général latin vulgaire, trois genres, dont le neutre, et qui puise ses étymons, au besoin moyennant quelques ajustements ponctuels (« 〈fiddled with〉 classical Latin », Buchi 2010, 2), dans les dictionnaires latins (« méthode de la pioche », Chambon 2010, 65).

\subsection{Le tournant protoroman}

Mais lors d'un des Ateliers DÉRom semestriels, durant lesquels l'équipe se rencontre pour discuter des articles et prendre des décisions de principe, la question du neutre se recommanda à l'attention de l'équipe. C'est en effet à l'occasion du $5^{\mathrm{e}}$ Atelier DÉRom, qui se tint les 9/10 juillet 2010 à Sarrebruck, que l'article */'ali-u/ (cf. Reinhardt 2010-2014 in DÉRom s.v.) fournit l'occasion de discuter pour la première fois de façon approfondie le statut du neutre au sein du projet.

La discussion convergea sur le constat que l'étiquetage comme neutres de substantifs comme */'ali-u/ ou */'lakt-e/ sur la seule base de l'appartenance au neutre de leurs corrélats du latin écrit de l'Antiquité était contraire à la philosophie du projet, fondée sur la reconstruction - phonologique et sémantique, mais aussi morphologique - des étymons. Il nous apparut en effet que si le latin permettait facilement d'attribuer à l'ancêtre des parlers romans un neutre, la reconstruction linguistique, utilisée seule, ne le pouvait pas : en effet, les idiomes romans possèdent des formes distinctes pour le masculin et le féminin, mais dans les quelques cas, dont le roumain est le plus massif (cf. Windisch 1973 ; Livescu 2008, 2647-2648), où ils possèdent un troisième genre, celui-ci est construit avec les formes de masculin et de féminin. Plusieurs langues romanes connaissent en effet des substantifs qui ont un genre au singulier et un autre au pluriel (le plus souvent le pluriel est féminin, avec des alternances du type d'it. braccio m.sg. braccia f.pl. 'bras'), et cette classe de noms constitue même une catégorie productive en roumain, qui le considère donc comme un troisième genre (appelé souvent ambigène). Mais les parlers romans n'utilisent jamais de morphèmes flexionnels spécifiques pour ces substantifs, ce qui nous amenait à conclure que nous ne pouvions pas, sur la seule base de formes masculines et féminines, reconstruire un genre qui aurait légitimement pu s'appeler neutre, sur le modèle du neutre latin ; une partie de l'assemblée estimait même que la reconstruction ne permettait pas de mettre ce genre du roumain en rapport de descendance directe avec la protolangue. 
Les membres du DÉRom réunis à Sarrebruck décidèrent alors, par précaution, d'adopter une terminologie plus descriptive et de remplacer le terme de neutre par celui d'ambigène pour caractériser le troisième genre du roumain - et, par voie de conséquence, celui de l'ancêtre commun des langues romanes, le protoroman - et de réserver le terme de neutre aux données du latin écrit. Nous retrouvions ainsi, comme nous devions nous en apercevoir ensuite, l'analyse de Hall, pour qui ces « ambigènes » ne peuvent dans aucune langue romane, et pas non plus en protoroman, être dits constituer un genre «neutre » séparé (Hall 1983, 25).

\subsection{La solution zurichoise}

Mais cette réduction de nos ambitions n'était pas destinée à s'installer durablement. À l'occasion du XXVI ${ }^{\mathrm{e}}$ Congrès de linguistique et de philologie romanes, qui eut lieu en 2010 à Valence, les membres du DÉRom prirent connaissance, à travers une communication présentée à trois voix (Faraoni/Gardani/Loporcaro 2013), des importants travaux que l'École zurichoise autour de Michele Loporcaro avait consacrés à la problématique du genre. Des contacts fructueux entre les deux groupes de recherche furent initiés et donnèrent lieu, dans un premier temps, à une conférence prononcée par Tania Paciaroni dans le cadre du Séminaire de l’ATILF à Nancy (Paciaroni 2011).

L'originalité de l'approche zurichoise consiste à sortir, pour l'analyse des genres dans les langues romanes, du cadre étroit des langues standardisées pour s'intéresser aux données dialectales, ce en quoi elle rejoint une particularité du projet DÉRom (cf. Andronache 2013). Or, certains dialectes italiens centro-méridionaux présentent ( $\mathrm{au}$ moins) un genre neutre du type target gender dans le sens de Corbett (1991, 151), c'est-à-dire des neutres pourvus de morphèmes flexionnels dédiées, du type latin classique ou allemand (Loporcaro/Paciaroni 2011 ; cf. aussi Faraoni 2016). Cette situation impose une analyse de ces variétés comme possédant un véritable neutre, fonctionnel, et non de simples occurrences isolées de substantifs au fonctionnement atypique, ainsi que la reconnaissance d'une continuité historique entre cette situation et le neutre latin.

Ces travaux de l'École zurichoise nous aidèrent d'abord à mieux poser le problème et ensuite à trouver des critères à mettre en œuvre pour sa solution. En effet, nous avions commencé par mal poser le problème, en interprétant comme deux genres différents ce qui n'était que deux moyens de marquer ce genre; nous n'étions ainsi pas loin de tomber dans un débat nominaliste. En appelant genre autonome celui qui est pourvu de marques propres et genre non 
autonome celui qui, comme le roumain, combine d'une manière différente des marques présentes dans les autres genres, nous étions en mesure de mieux situer le débat. Prenant appui sur ces considérations, les membres du DÉRom réunis à Nancy à l'occasion du $8^{\mathrm{e}}$ Atelier DÉRom en juin 2012 décidèrent de renoncer à la catégorisation en substantifs ambigènes et de revenir à la solution initiale, à savoir de retenir le terme de neutre tant pour le roumain que pour le protoroman.

Nous partons donc désormais du principe qu'il y a lieu, pour certaines variétés romanes au moins, de poser l'existence d'un neutre autonome, issu de la protolangue sans solution de continuité. Cela conduit immédiatement à conclure de son existence en protoroman. Le travail du comparatiste reviendra par conséquent à déterminer, pour chaque unité lexicale traitée, si elle remonte à un substantif possédant ce genre; la disparition du caractère productif de cette catégorie grammaticale conduira à analyser comme neutres des substantifs ne présentant plus que quelques traces de leur ancien statut.

\section{Reflet lexicographique de la solution adoptée}

\subsection{Cas général : étymons neutres pourvus de corrélats neutres}

Le DÉRom contient pour le moment seize articles publiés pour lesquels il juge nécessaire de reconstruire un neutre: */'agr-u/ (Alletsgruber 2014/2015 in DÉRom s.v.), */'ali-u/ (Reinhardt 2010-2014 in DÉRom s.v.), */' $\beta$ in-u/ (Delorme 2011-2015 in DÉRom s.v.), */'dJl-u/ (Morcov 2014-2016 in DÉRom s.v.), */'фamen/ (Buchi/González Martín/Mertens/Schlienger 2012-2015 in DÉRom

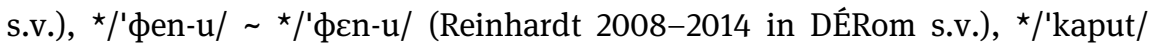
(Schmidt/Schweickard 2015/2016 in DÉRom s.v.), */'kasi-u/ (Delorme 2011-2014 in DÉRom s.v.), */'lakt-e/ (Delorme 2011-2014 in DÉRom s.v.), */'lumen/ (Georgescu 2014-2016 in DÉRom s.v.), */'must-u/ (Delorme 2011-2016 in DÉRom s.v.), */pek'k-at-u/ (Ney/Maggiore 2014/2015 in DÉRom s.v.), */'pes-u/ (Morcov 2014 in DÉRom s.v.), */'preti-u/ (Groß 2015 in DÉRom s.v.), /'rap-u/ (Delorme 2013/2014 in DÉRom s.v.) et */'unkt-u/ (Videsott 2012-2015 in DÉRom s.v.).

Les arguments principalement utilisés pour rendre probable la catégorie grammaticale « neutre » d'une unité lexicale en protoroman sont d'une part son appartenance au genre neutre en roumain, d'autre part une alternance de genre dans d'autres variétés romanes. La typologie des unités traitées va du douteux (les cas où seul le roumain, ou pire seul le dacoroumain, atteste un neutre, et 
pour lesquels le DÉRom considère que la reconstruction d'un neutre n'est pas assurée) au très assuré, lorsqu'en plus de la présence d'un neutre en roumain, plusieurs idiomes romans présentent des vestiges d'un ancien neutre.

Le cas de doute maximal est représenté pour l'instant par le seul article */'ßad-u/ (Alletsgruber 2011-2016 in DÉRom s.v.), dont le lemme contient l'indication de partie du discours «s.[n. ou m.] », catégorisation insolite qui trouve son explication dans le commentaire :

« Du point de vue morphosyntaxique, la comparaison reconstructive ne permet pas de trancher avec sûreté entre un étymon neutre (recommandé par le genre neutre du cognat dacoroumain, $c f$. ci-dessus I.) et un étymon masculin (recommandé par celui des autres cognats, $c f$. ci-dessus II. et III.). Étant donné le caractère récessif du neutre dans les langues romanes, on aurait tendance à reconstruire un neutre, mais dans la mesure où ce genre est toujours productif en dacoroumain et que l'on ne dispose d'aucun cognat suddanubien, il existe un léger doute. Le corrélat du latin écrit de I., uadum, -i s.n. 'gué', est connu durant toute l'Antiquité (dp. Plaute [* ca $254-+184$ ], OLD), celui de II., uadus, -i s.m. 'id.', est attesté sporadiquement entre Varron $\left({ }^{\star} 116-\dagger 27\right)$ et Fronton $\left({ }^{\star} c a 100-\dagger c a\right.$ 170 , tous les deux OLD). La reconstruction d'un neutre originel, que la seule méthode comparative ne permet pas d'établir de façon définitive, trouve donc dans le corrélat de la langue écrite un appui solide » (Alletsgruber 2011-2016 in DÉRom s.v. */'ßad-u/).

Cet article est représentatif d'un cas de figure que nous avons encore rarement rencontré, mais qui présente un défi épistémologique intéressant en raison de l'impossibilité d'aller au terme de la reconstruction (pour plus de détails, cf. Maggiore/Buchi 2014, 317-320 [« Le latin écrit comme bénédiction »]).

À l'autre bout de l'échelle, l'article */'lakt-e/ présente un cas extrêmement favorable à la reconstruction, et qui a d'ailleurs déjà été exploité dans ce sens par de Dardel (1974). En effet, le caractère héréditaire du genre neutre de protorom. */'lakt-e/ est rendu très assuré par sa présence dans tous les dialectes sub-danubiens du roumain (en plus du dacoroumain). Il est encore confirmé par la configuration spéciale qui voit les parlers romans se répartir les trois genres. En dehors des variétés roumaines, seules à conserver le neutre, le féminin est présent d'une part dans une aire située à cheval entre Gallia et Iberia, et allant du languedocien occidental à l'asturien oriental et à l'espagnol, d'autre part en Vénétie, le masculin dans tous les autres parlers romans, son aire étant rendue discontinue par celle du féminin. Cette configuration, ainsi que le parallèle des autres cas discutés par de Dardel, conduit Jérémie Delorme, l'auteur de l'article */'lakt-e/, à une analyse en trois couches : I. neutre, II. masculin, III. féminin, se succédant chronologiquement. Cette analyse se trouve en accord avec les données latines, auxquelles elle donne plus de poids. Le latin connaît en effet l'usage du neutre, ancien et usuel, celui du masculin, un peu 
plus tardif et moins fréquent, et celui du féminin, tout à fait tardif $\left(5^{\mathrm{e}} / 6^{\mathrm{e}}\right.$ siècles) et rare.

Nous observons ainsi, dans cet exemple et dans les autres articles pour lesquels le DÉRom reconstruit un neutre, une forte similarité entre le résultat de la reconstruction et les données latines, y compris lorsque celles-ci sont complexes, ce qui nous rassure quant à la validité de nos reconstructions.

\subsection{Exemple d'un étymon neutre dépourvu de corrélat neutre}

On vient de voir que le cas le plus répandu est celui constitué par des étymons neutres du DÉRom pourvus d'un corrélat en latin écrit de l'Antiquité qui présente lui aussi le genre neutre. Mais il va de soi que la reconstruction comparative n'a pas besoin de la présence d'un corrélat, dont elle est logiquement indépendante. Nous allons donc terminer par un exemple d'étymon neutre que nous avons été amenés à reconstruire dans le cadre du DÉRom qui ne présente pas de corrélat neutre en latin écrit de l'Antiquité: l'ancêtre commun de dacoroum. foame s.f. 'faim', istroroum. fóme, aroum. foame, dalm. 'fum', istriot. $\ulcorner$ fan $\urcorner$, it. fame, sard. 'famene $\urcorner$ m., frioul. fam f., lad. fam, romanch. fom etc. (cf. Buchi/González Martín/Mertens/Schlienger 2015).

Le $\mathrm{REW}_{3}$ et le FEW n'assortissent pas leur lemme d'une indication explicite concernant le genre, mais les principes axiomatiques qui gouvernent ces deux opera magna de l'étymologie romane amènent à y voir, sur le modèle du latin écrit, des féminins :

- « 3178. fames [s.f.] 'Hunger', 2. *famine » $\left(\mathrm{REW}_{1} 1911-\mathrm{REW}_{3} 1935\right)$; $^{2}$

- «fames [s.f.] hunger » (von Wartburg 1931 in FEW 3, 406a).

La nomenclature provisoire du DÉRom ne s'écartait pas de ces vues, puisqu'elle prévoyait un article */'фam-e/ étiqueté "substantif féminin ». Lors de la rédaction de cet article, réalisée dans le cadre du European Master in Lexicography (EMLex, cf. 〈http://www.atilf.fr/emlex〉), nous pensions dans un premier temps maintenir ce lemme, tout en prévoyant, selon le modèle du $\mathrm{REW}_{3}$ et du FEW, ${ }^{3}$ un sous-lemme */' $\phi$ amin-e/ s.f. comme ancêtre commun direct d'un petit groupe de lexèmes, notamment gasc. hame et esp. hambre. Au cours de la

$2 \mathrm{REW}_{1}$ porte ${ }^{\star}$ famine.

3 Après avoir transféré sous */' $\phi a m-e /$ port. fome, que le REW 3 et le FEW classaient par erreur sous *famine (cf. */'фamen/ n. 6). Par ailleurs, nous n'avons pas retrouvé «arum. foamine » que le $\mathrm{REW}_{3}$ cite sous 2 . Pour ce qui est de sard. 'famene ${ }^{7}$, cf. ci-dessous. 
rédaction, deux autres prototypes, pressentis dans un premier temps comme des sous-lemmes d'un tel article intitulé */'фam-e/, s'imposèrent : d'une part */фa'min-a/ s.f., reconstruit à partir de fr. famine et ses cognats ligurien, piémontais, romanche, francoprovençal, occitan et catalan, d'autre part */'фamit-e/ s.f., limité au protoroumain et reconstruit à partir de cognats dacoroumain et aroumain. Jusque là, tous nos étymons directs étaient féminins, et nous étions persuadés que l'étymon protoroman serait également féminin.

Ce qui fit pencher la balance en faveur d'un étymon neutre, ce sont les données sardes. En effet, il est impossible de rattacher sard. 'famene ${ }^{\urcorner}$s.m. 'faim ; famine', comme le proposaient le $\mathrm{REW}_{3}$ et le FEW, au type qui se présente dans le DÉRom sous la forme */'фamin-e/. D'abord pour des raisons phonologiques : la voyelle finale [-e] dans 'famene ${ }^{\urcorner}$ne remonte pas à la protoforme : il s'agit d'une simple voyelle paragogique (cf. Wagner 1984, 101-102 et */'фamen/ n. 18) ; la typisation, si elle est étymologisante, devrait donc être `famen $\urcorner$ et non pas ${ }^{\ulcorner}$famene $\urcorner$. Et comme ${ }^{\star} /$-e/ final se maintient en sarde (MeyerLübke 1890, vol. 1, $262 \S 306$; Wagner 1984, 69-74), cette unité lexicale ne peut pas continuer */'фamin-e/. Il s'y ajoute une particularité morphosyntaxique : à la différence de l'ensemble des autres cognats réunis dans cet article, tous féminins, sard. 'famen $\urcorner$ est de genre masculin. Ces deux critères incitent à reconstruire dans un premier temps protosard. */'фamen/ s.m., ce qui porte donc à cinq les étymons directs reconstruits : */'фam-e/ (s.f.), */'фamen/ (s.m.),

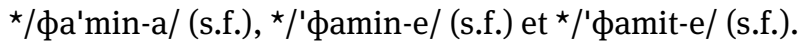

L'étape suivante consiste à assigner, en convoquant notamment des critères aréologiques, à chacune de ces protoformes une époque de création, afin de déterminer leur point d'origine commun. Cette analyse fait apparaître que

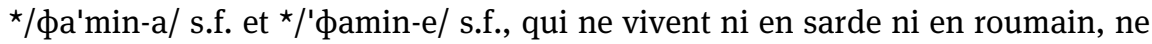
remontent pas plus haut que l'état de langue que l'on pourrait appeler, suite à Hall (1950, 25 : «Proto-Italo-Western Romance »), le protoroman continental italo-occidental : ce sont les deux innovations les plus récentes. On écartera aussi */'фamit-e/ s.f., que l'on ne peut pas assigner à une strate plus ancienne que le protoroumain. Les deux protoformes restantes occupent chacune une des branches du premier nœud de l'arbre phylogénétique de l'étymon de la protolangue : */'фam-e/ s.f., qui manque en sarde, mais vit en roumain et dans un grand nombre de parlers italo-occidentaux, est assignable au protoroman continental, tandis que */'фamen/ s.m. relève du protosarde.

Dès lors, trois hypothèses sont envisageables pour la reconstruction de la protoforme en protoroman commun (ou protoroman stricto sensu) :

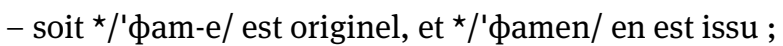

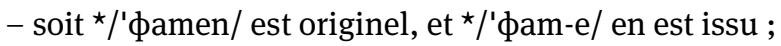


- soit */'фam-e/ et */'фamen/ représentent tous les deux des évolutions d'un ancêtre commun qui n'a ni la forme */'фam-e/ ni la forme */'фamen/.

La première hypothèse peut être exclue, car il n'y a aucun processus linguistique, qu'il soit phonétique ou morphologique, qui expliquerait le passage de */'фam-e/ à */'фamen/. La troisième hypothèse ne s'envisage pas non plus, car il n'existe pas de phonème autre que $\star / n /$ dont l'issue pourrait être d'une part /n/, d'autre part zéro. On en conclura - avec l'hypothèse 2 - que */'фamen/ est originel et que */'фam-e/ en est issu.

Maintenant que nous disposons du signifiant de l'étymon protoroman sachant qu'à travers des procédures analogues, on lui aura attribué le signifié complexe 'faim ; famine ; désir' -, arrive le moment de se poser la question, centrale dans notre contexte, du genre qu'il convient de lui attribuer.

Pour ce faire, on adopte la posture de la reconstruction interne pour s'interroger sur la plausibilité du trait reconstruit à l'intérieur du système linguistique protoroman. Or, autant un substantif masculin dont le signifiant est */'фamen/ est possible en protosarde, autant en protoroman, un substantif se terminant par $\star /$-'amen/ relève obligatoirement du genre neutre : il s'insère dans la série */e'samen/ s.n. 'essaim', */'gramen/ s.n. 'herbe', */'stramen/ s.n.

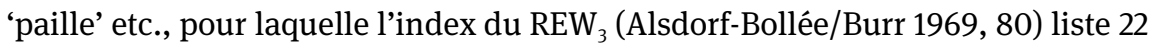
items. $^{4}$

Voilà le raisonnement qui nous a amenés à reconstruire le genre neutre pour l'étymon de fr. faim et de ses cognats. Par ailleurs, l'hypothèse d'un neutre originel trouve trois confirmations à l'intérieur même du scénario reconstruit.

Premièrement, il est significatif que parmi les deux issues directes de

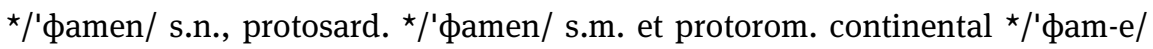
s.f., l'une présente le genre masculin, l'autre le genre féminin : voilà les deux solutions possibles que les parlers romans autres que roumains avaient à leur disposition pour gérer le genre récessif hérité de la protolangue. Inversement, identifier comme originel un trait irrégulier relève de la méthode comparative la plus classique.

Deuxièmement, le type */фa'min-a/ véhicule une issue indirecte du neutre : nous y voyons le résultat d'une remorphologisation entraînant un changement d'accentuation (peut-être par attraction du suffixe $\star /-' i n-a /$ ) du pluriel

4 Treize d'entre eux (aeramen, exāmen, forāmen, gramen, laetāmen, lèvāmen, lügāmen, lĭgnāmen, *materiāmen, mĕdĭcāmen, pŭllāmen, stamen, stramen) sont dotés d'un corrélat de genre neutre en latin écrit de l'Antiquité ; les neuf autres ( ${ }^{\star}$ allěvāmen, *aramen, *cŏriāmen, ${ }^{\star}$ feramen, ${ }^{*}$ funāmen, ${ }^{\star}$ inč̌sāmen, ${ }^{\star}$ lorāmen, ${ }^{\star}$ pěllāmen et ${ }^{*}$ pìtāmen) ne sont pas attestés en latin de l’Antiquité (Ø TLL ; Ø OLD). 
*/'фamin-a/ de l'étymon originel. Cela signifie que le neutre */'фamen/ a perduré (comme variante de $\star / ' \phi a m-e /$ ) au-delà de la période pour laquelle nous pouvons le reconstruire sur la base du seul singulier (protoroman commun), ce qui n'a rien de choquant quand on tient compte du témoignage des parlers italiens centro-méridionaux, qui invitent à reconstruire une catégorie neutre de type "genre autonome" pour le protoroman continental italo-occidental.

Et troisièmement, une fois qu'un étymon neutre se trouve au sommet de la reconstruction, la pléthore de protoformes secondaires s'explique aisément. D'une part, le passage de */'фamen/ à */' $\phi a m-e /$ relève de la tendance générale au passage au féminin des substantifs de la troisième déclinaison, en particulier de ceux en $\star / '-$-men/, que la réduction phonétique $* /-e n />\star /-e /$ qui a régulièrement frappé toute la Romania continentale a entraînés dans le champ d'attraction de la flexion en */-e/ de type */'ment-e/. D'autre part, */' $\phi a m i n-e /$ et */' $\phi$ amit-e/ - et, quand on y réfléchit, aussi $\star / \phi a ' m i n-a /$ - doivent leur existence à différentes sortes d'alignement sur des types flexionnels présentant une forme de l'accusatif distincte de celle du nominatif, ce qui concorde bien avec l'hypothèse d'un changement de genre.

\section{Conclusion}

En guise de conclusion, voici un rappel des quatre points qui nous semblent se dégager de ces lignes :

- Les résultats de recherche de l'École de Zurich ont constitué un apport déterminant dans la réflexion autour du traitemement du neutre dans le projet DÉRom.

- Le détour par une catégorisation erronée a contribué à aiguiser les outils d'analyse conceptuelle du projet.

- Dans la majorité des cas, la méthode comparative appliquée à l'étymologie romane ne fait que confirmer le genre neutre des étymons que la méthode traditionnelle avait déjà tenu comme acquis.

- En revanche, dans certains rares cas, l'application de la méthode comparative au lexique roman permet, en raison de la «rupture avec la conception littérario-centrique » (Chambon 2014, 154) qu'elle véhicule nécessairement, de reconstruire des étymons neutres qui échappaient à la méthode traditionnelle. 
En raison de son caractère pionnier, le projet DÉRom est caractérisé par une démarche de va-et-vient continuel entre travail rédactionnel et perfectionnement de la méthode. En effet, le DÉRom découvre des problèmes méthodologiques souvent inédits - et doit les résoudre par la discussion - en avançant ; cette option stratégique retarde le projet, mais l'enrichit aussi considérablement.

\section{Bibliographie}

Alsdorf-Bollée, Annegret/Burr, Isolde, Rückläufiger Stichwortindex zum Romanischen Etymologischen Wörterbuch, Heidelberg, Winter, 1969.

Andronache, Marta, Le statut des langues romanes standardisées contemporaines dans le «Dictionnaire Étymologique Roman » (DÉRom), in : Emili Casanova Herrero/Cesáreo Calvo Rigual (edd.), Actas del XXVI Congreso Internacional de Lingüística y de Filología Románicas (Valencia 2010), vol. 4, Berlin/New York, De Gruyter, 2013, 449-458.

Buchi, Éva, Where Caesar's Latin does not belong : a comparative grammar based approach to Romance etymology, in : Charlotte Brewer (ed.), Selected Proceedings of the Fifth International Conference on Historical Lexicography and Lexicology held at St Anne's College, Oxford, 16-18 June 2010, Oxford, Oxford University Research Archive 〈http://ora.ox.ac.uk/objects/uuid\%3A237856e6-a327-448b-898c-cb1860766e59〉, 2010.

Buchi, Éva/González Martín, Carmen/Mertens, Bianca/Schlienger, Claire, L'étymologie de FAIM et de FAMINE revue dans le cadre du DÉRom, Le français moderne 83 (2015), 248-263.

Campbell, Lyle, Historical Linguistics. An Introduction, Cambridge, MIT Press, ${ }^{3} 2013$ [11998].

Chambon, Jean-Pierre, Pratique étymologique en domaine (gallo)roman et grammaire comparée-reconstruction. À propos du traitement des mots héréditaires dans le «TLF » et le « FEW », in : Injoo Choi-Jonin/Marc Duval/Olivier Soutet (edd.), Typologie et comparatisme. Hommages offerts à Alain Lemaréchal, Louvain/Paris/Walpole, Peeters, 2010, 6-175.

Chambon, Jean-Pierre, Réflexions sur la reconstruction comparative en étymologie romane : entre Meillet et Herman, in : Gleßgen, Martin/Schweickard, Wolfgang (edd.), Étymologie romane : objets, méthodes et perspectives, Strasbourg, ÉLiPhi, 2014, 141-159.

Corbett, Greville G., Gender, Cambridge, Cambridge University Press, 1991.

Dardel, Robert de, Une analyse spatio-temporelle du roman commun reconstruit (à propos $d u$ genre), in : Alberto Vàrvaro (ed.), XIV Congresso internazionale di linguistica e filologia romanza, Napoli 15-20 aprile 1974, vol. 14/2, Naples/Amsterdam, Macchiaroli/Benjamins, 1976, 75-82.

DÉRom = Buchi, Éva/Schweickard, Wolfgang (dir.), Dictionnaire Étymologique Roman (DÉRom), Nancy, ATILF, «http://www.atilf.fr/DERom>, 2008-.

Faraoni, Vincenzo, Manifestazioni del neutro italo-romanzo nella documentazione notarile altomedievale, in : Éva Buchi/Jean-Paul Chauveau/Jean-Marie Pierrel (edd.), Actes du XXVII ${ }^{e}$ Congrès international de linguistique et de philologie romanes (Nancy, 15-20 juillet 2013), vol. 1, Strasbourg, ÉLiPhi, 2016, 381-395. 
Faraoni, Vincenzo/Gardani, Francesco/Loporcaro, Michele, Manifestazioni del neutro nell'italoromanzo medievale, in : Emili Casanova Herrero/Cesáreo Calvo Rigual (edd.), Actas del XXVI Congreso Internacional de Lingüística y de Filología Románicas (Valencia 2010), vol. 2, Berlin/New York, De Gruyter, 2013, 171-182.

FEW = Wartburg, Walther von et al., Französisches Etymologisches Wörterbuch. Eine darstellung des galloromanischen sprachschatzes, 25 vol., Bonn/Heidelberg/LeipzigBerlin/Bâle, Klopp/Winter/Teubner/Zbinden, 1922-2002.

Hall, Robert A. Jr., The Reconstruction of Proto-Romance, Language 26 (1950), 6-27.

Hall, Robert A. Jr., Comparative Romance Grammar. Volume III : Proto-Romance Morphology, Amsterdam/Philadelphie, Benjamins, 1983.

Livescu, Michaela, Histoire interne du roumain : morphosyntaxe et syntaxe, in : Gerhard Ernst et al. (edd.), Histoire linguistique de la Romania. Manuel international d'histoire linguistique de la Romania, vol. 3, Berlin/New York, De Gruyter, 2008, 2646-2692.

Loporcaro, Michele/Paciaroni, Tania, Four-gender systems in Indo-European, Folia Linguistica 45 (2011), 389-433.

Maggiore, Marco/Buchi, Éva, Le statut du latin écrit de l'Antiquité en étymologie héréditaire française et romane, in : Franck Neveu et al. (éd.), Actes du Congrès Mondial de Linguistique Française 2014 (Berlin, 19-23 juillet 2014), Paris, Institut de Linguistique Française, 2014, «http://dx.doi.org/10.1051/shsconf/20140801161), 313-325.

Meyer-Lübke, Wilhelm, Die Schicksale des lateinischen Neutrums im Romanischen, Halle, Karras, 1883.

Meyer-Lübke, Wilhelm, Grammaire des langues romanes, 4 vol., Paris, Welter, 1890-1906 [original allemand : 1890-1902].

OLD = Glare, Peter G. W. (ed.), Oxford Latin Dictionary, Oxford, Clarendon, 1968-1982.

Paciaroni, Tania, Genèse et développement de la catégorie du neutre, conférence prononcée dans le cadre du "Séminaire de l'ATILF », Nancy, ATILF, 15 avril 2011.

$\mathrm{REW}_{3}=$ Meyer-Lübke, Wilhelm, Romanisches Etymologisches Wörterbuch, Heidelberg, Winter, ${ }^{3} 1935\left[{ }^{1} 1911-1920\right]$.

TLL = Thesaurus Linguae Latinae, Leipzig/Stuttgart/Berlin/New York, Teubner/Saur/De Gruyter, 1900-.

Wagner, Max Leopold, Fonetica storica del sardo, Cagliari, Gianni Trois, 1984 [original allemand : 1941].

Windisch, Rudolf, Genusprobleme im Romanischen : das Neutrum im Rumänischen, Tübingen, Narr, 1973. 